\title{
CORONARY FLOW IN NEONATES WITH IMPAIRED INTRAUTERINE GROWTH
}

\author{
E.H. Aburawi ${ }^{1}$, P. Malcus ${ }^{2}$, A. Thuring ${ }^{2}$, V. Fellman ${ }^{3}$, E. Pesonen ${ }^{3}$ \\ ${ }^{1}$ Paediatric, United Arab Emirates University, Faculty of Medicine \& Health Sciences, Al-Ain, United Arab \\ Emirates, ${ }^{2}$ Obs. and Gynecology, ${ }^{3}$ Paediatrics, Skane University Hospital, Lund University, Lund, Sweden
}

Background: Subclinical myocardial injury has been reported in newborns with fetal weight below 2 standard deviations for gestational age. Our aim was to investigate whether impaired intrauterine growth affects cardiac function and coronary flow (CF).

Subjects and methods: Seventeen newborns with impaired intrauterine growth and fifteen age-matched healthy controls were enrolled in the study. Fetal growth was assessed by fetometry. Doppler velocimetry of the umbilical artery and maternal uterine arteries blood flow was assessed. Cardiac function and left anterior descending artery (LAD) coronary flow were measured by transthoracic Doppler echocardiography at one week of age.

Results: The mean growth deviation of the newborns from normal was $-2.5 \pm 0.2 \mathrm{SD}$. The left ventricular mass and left ventricular shortening fraction was similar in patients and controls. The mean LAD diameter was $0.99 \pm 0.1 \mathrm{~mm}$ in patients and $0.8 \pm 0.1$ in controls, $\mathrm{p}=0.002$. LAD flow velocity time integral $(\mathrm{VTI}) / \mathrm{min}$ correlated with left ventricular mass $(R=0.46, \mathrm{p}=0.0001)$ and with mitral peak E-wave velocity $(R=0.74$, $\mathrm{p}<0.01$ ). Impaired intrauterine growth was associated with increased peak flow velocity in diastole; $34.5 \pm 4$ $\mathrm{cm} / \mathrm{s}$ and $19 \pm 6 \mathrm{~cm} / \mathrm{s}$ in controls, $p=0.0001$ as well as increased CF; $37 \pm 7.3 \mathrm{ml} / \mathrm{min}$ and $8.2 \pm 3.0 \mathrm{ml} / \mathrm{min}$ in controls, $p=0.001$.

Conclusions: Coronary flow is significantly increased in neonates with impaired intrauterine growth. The left ventricular mass and systolic and diastolic functions remain normal. The clinical significance of the increase of $\mathrm{CF}$ is unclear but increased basal flow might lead to a decreased coronary flow reserve. 